\title{
Production of RPC gaps for the CMS upgrade
}

\author{
Sung Keun Park* \\ On behalf of the CMS collaboration \\ Korea Detector Laboratory, Korea University \\ Seoul, 136-701, Korea \\ E-mail: sungpark@korea.ac.kr
}

The CMS collaboration intends to improve the muon trigger efficiency in the forward region. In order to achieve this goal, 144 new Resistive Plate Chambers (RPCs) will be installed as a 4th layer in the endcap system, to trigger high momentum muons from the proton-proton interaction. In this paper, we present the detailed procedures used in the production of the CMS RPC gas gaps adopted in the CMS upgrade. Quality assurance is enforced in order to maintain the quality of RPC gas gaps at a similar level as for the previous CMS endcap RPC chambers. Both the production procedures and the quality assurance are mature, and effective for the mass production of these RPC gas gaps.

XI workshop on Resistive Plate Chambers and Related Detectors - RPC2012,

February 5-10, 2012

INFN Laboratori Nazionali di Frascati Italy

${ }^{*}$ Speaker.

$\dagger$ I acknowledge the support of the Korean Research Foundation, and Korea University. 


\section{Introduction}

The CMS experiment emphasizes the detection and identification of muons [1].

At nominal running conditions, every $25 \mathrm{~ns}$ some 1000 particles emerge from the interaction point into the CMS spectrometer. In less than $3 \mu \mathrm{s}$ a first level trigger has to reduce this rate to 100 $\mathrm{kHz}$ without losing potentially interesting collisions requiring further analysis.

RPCs are included in the muon trigger and provide redundancy for CSCs and DTs. Six concentric layers of chambers are used in the barrel part, while four layers have been foreseen in total for the end caps to cover a rapidity up to $\eta=2.1$. However, only 3 layers per end cap were installed with a limited rapidity coverage up to $\eta=1.6$. Therefore, the completion of the forward RPC system to 4 layers per end cap with a coverage up to $\eta=1.6$ is a priority. The basic structure of the CMS double gap RPC is shown in Fig. 1.

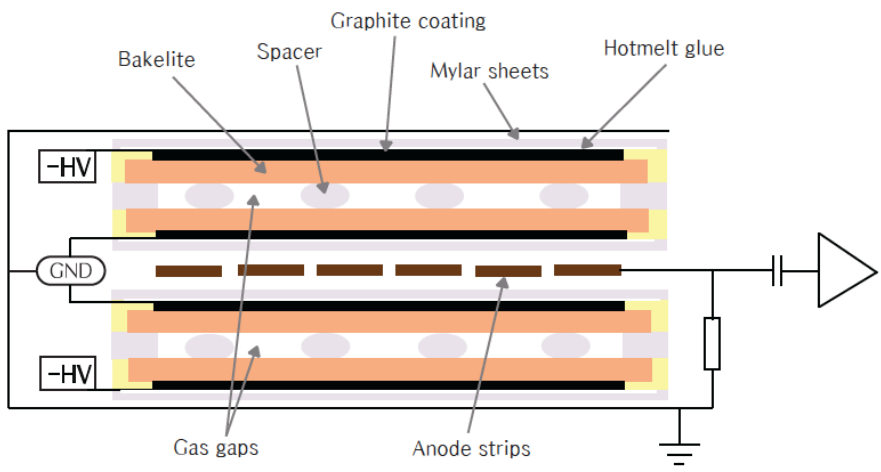

Figure 1: Cross sectional view of double gap RPC.

For the RPCs to provide an efficient muon trigger to CMS, the gas gaps must meet the CMS requirements for RPCs as shown in Table 1. To produce the gas gaps which meet these performance requirements, one should build the gaps with two most important considerations in mind: an overall mechanical stability and an uniform electric field over the active gas volume. In this paper, we describe each step in detail following the same technology that Korea Detector Laboratory (KODEL) developed for the forward RPCs which have been already installed and operational since the turn on the LHC. The high quality of the CMS double gap RPC is well captured in Fig. 2 as in [2].

\section{Production procedure}

The Bakelite sheets are produced, cut and cleaned in Italy and delivered to KODEL [?]. Once the bakelite sheets are prepared for the gap production as shown in Fig. 3, the next step is the production of the electrodes. A thin layer of graphite is coated on the bakelite sheet by a silkscreen process. The thickness of the graphite layer is well maintained by the $20 \mu \mathrm{m}$ thick silk screen mesh. The surface resistivity of the graphite layer ranges from 100 to $250 \mathrm{k} \Omega / \mathrm{square}$. The measured graphite surface resistivity for 50 samples is shown in Fig. 4. 

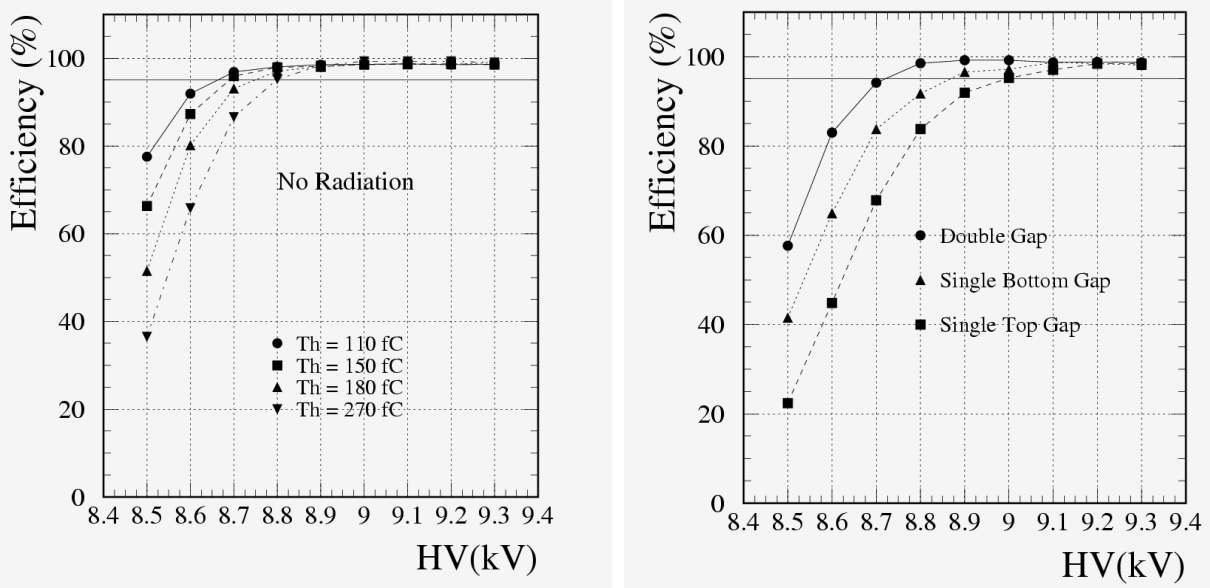

Figure 2: The efficiencies of the RPC in terms of charge thresholds (left), and number of gaps (right).

Table 1: CMS RPC requirements.

\begin{tabular}{ll}
\hline Parameters & Allowable ranges \\
\hline Efficiency & $>95 \%$ \\
Time resolution & $\leqslant 1 \mathrm{~ns}$ \\
Average cluster size & $\leqslant 2 \mathrm{strips}$ \\
Rate capability & $2 \mathrm{kHz} / \mathrm{cm}^{2}$ \\
Mean avalanche charge & $2.5-5 \mathrm{pC}$ \\
\hline
\end{tabular}

Once the graphite layer has dried, a protective insulator sheet made of Mylar is pasted on the graphite layer using a thin film of a hot adhesive based on ethylene vinyl acetate (EVA).
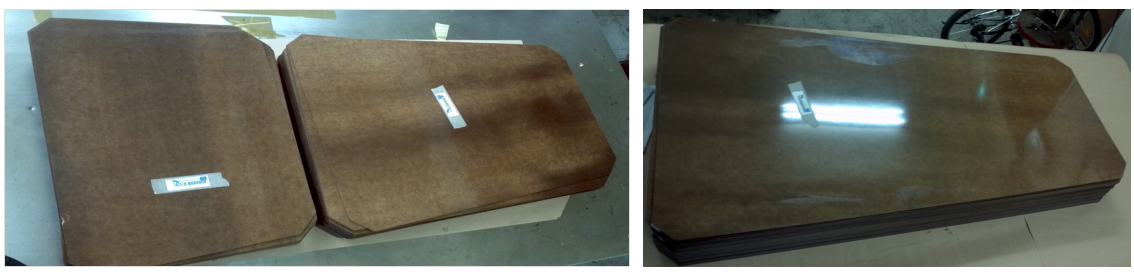

Figure 3: Bakelite sheets that are cut for the RE4/2 top narrow and top wide gaps (left), and bottom gaps (right).

Once the electrodes are prepared, the gas gaps are assembled with two electrodes and spacers. Coin shaped spacers are placed between the two electrodes to form a uniform separation of $2 \mathrm{~mm}$ between the electrodes, and the edge of the gas gap is sealed with an edge strip. The mechanical support of the gas gap is provided by the coin shaped spacers placed $10 \mathrm{~cm}$ apart from each other in two orthogonal directions. The edge strip that seals the edge of the gas gap makes the gas volume leak-tight. The spacers and edge strips are placed in pre-determined locations guided by a special 


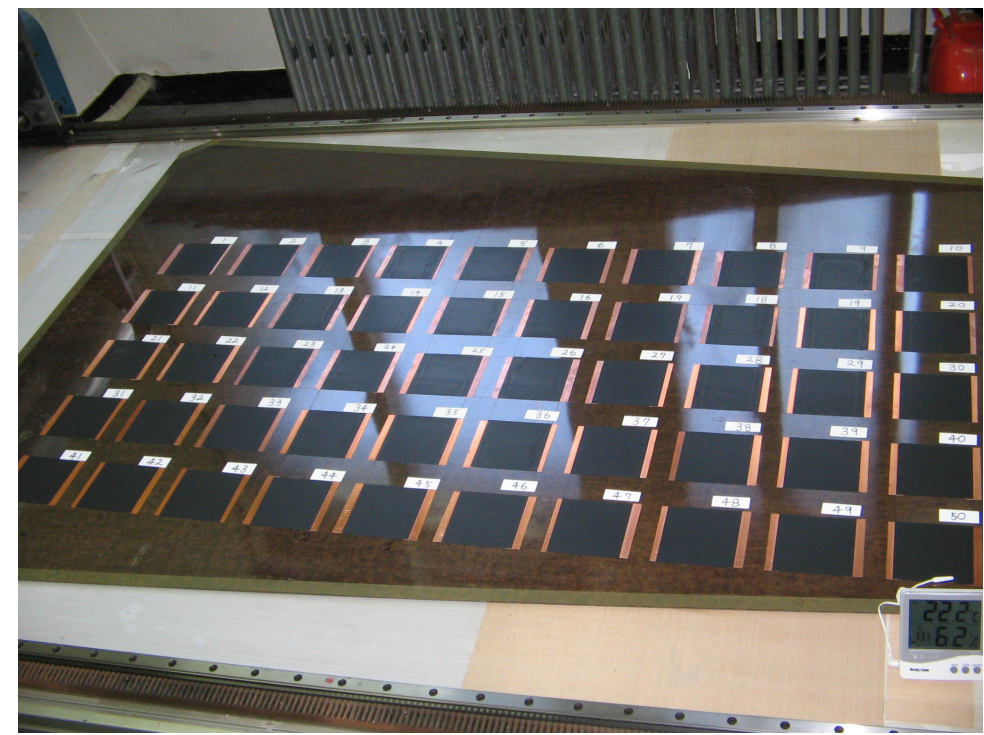

Figure 4: Arrangement of 50 samples of bakelite pieces to check the surface resistivity.

jig and are bonded to the electrodes using an epoxy with a curing time of 24 hours.

To achieve the maximum bonding strength between the spacers and electrodes, the gaps are placed in a device where an air pressure of $30 \mathrm{hPa}$ is applied over the entire surface of the gas volume.

After 24 hours of hardening, the mechanical stability of the gas gap is checked by a test to see the stability of the bonding and a test of the gas tightness of the gas volume. For the mechanical stability test, the gas volume is over-pressured with $20 \mathrm{hPa}$. For a gas gap to be qualified, any loss of the applied pressure should be less than $0.2 \mathrm{hPa}$ during a 10 minute period. In order for the gas gaps to pass the test, no spacer should lose its bonding strength between two electrodes during the $20 \mathrm{hPa}$ over-pressure application.

All these mechanical tests are carried out by a robot built at KODEL as shown in Fig. 5. The test results are stored in a CMS central ORACLE data base.

Once the gas gap passes the mechanical stability test, its gas volume is treated with linseed oil. It turns out that the polymerized linseed-oil layer on the surface of the bakelite inside the RPC reduces the spurious noise in the avalanche mode of operation. However, this oiling procedure needs a cautious preparation due to the pressure built up by the linseed oil filling the gas gap. If this pressure is greater than $20 \mathrm{hPa}$, the gas gap will likely to lose its epoxy bonding strength.

The CMS gas gaps have six different sizes and shapes. They are three gaps in each RE4 chamber as shown in Fig. 3. Accommodating this variety of physical dimensions to the oil coating facility is a difficult task due to the different orientations of the gas inlets and outlets depending on the shape of the gas gaps. Therefore, prior to the application of the linseed oil to the final batches 


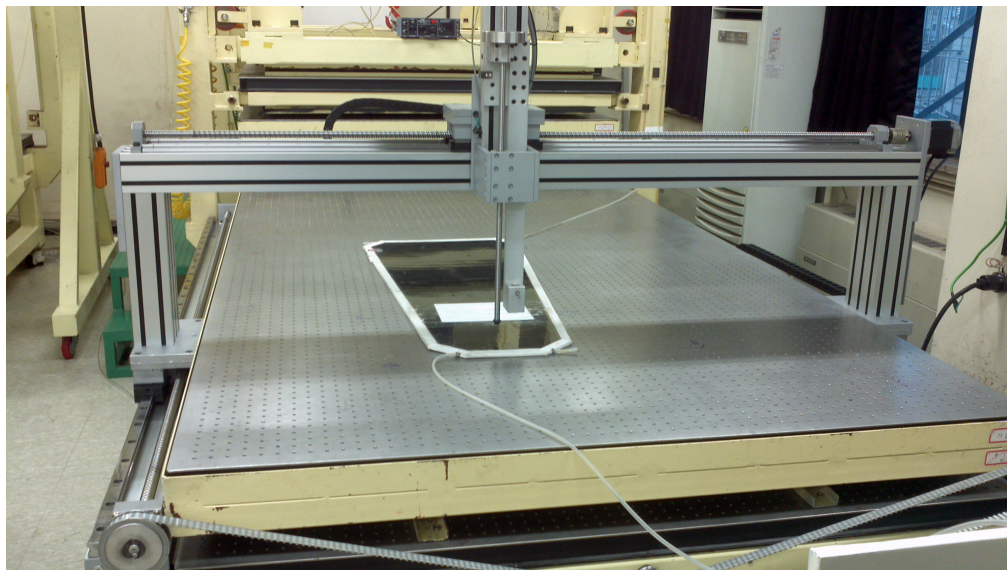

Figure 5: KODEL robot performing the pressure test.

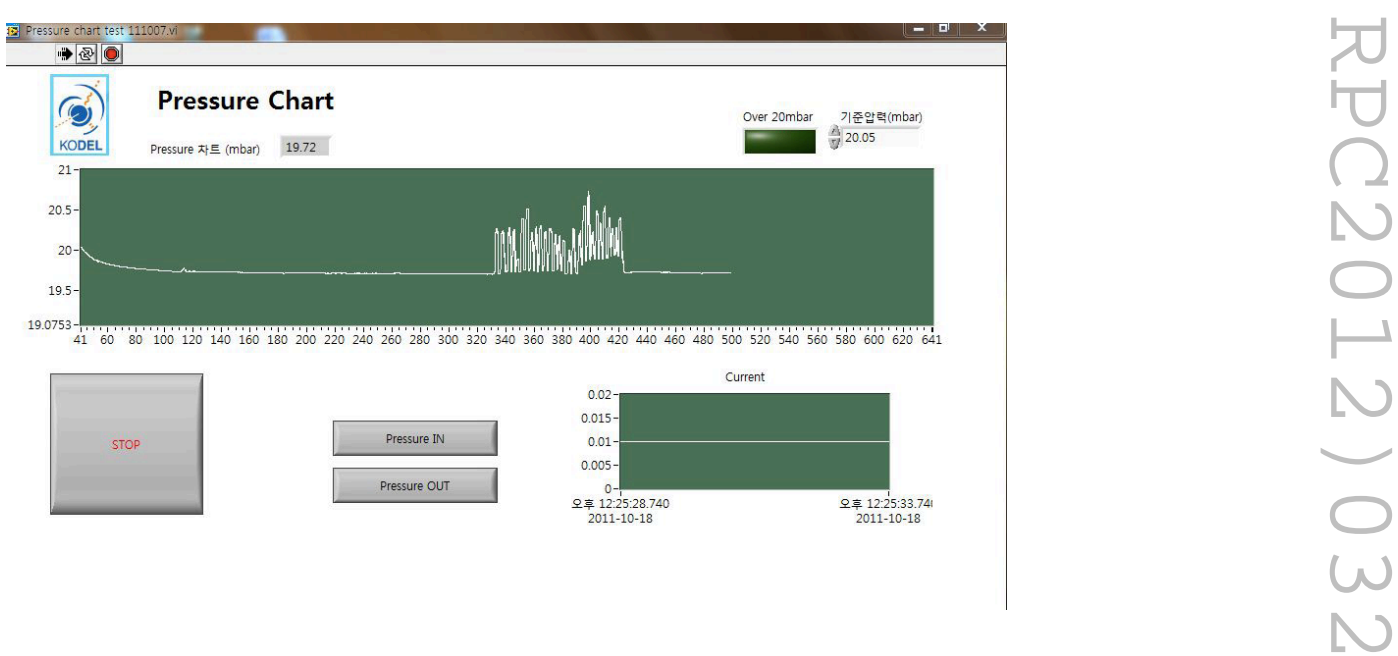

Figure 6: Test results of the pressure test.

of the gas gaps, a simulation with test gas gaps was performed.

Once the gas volume is treated with linseed oil, the current drawn by the gas gap is measured as this is a good indicator of any electrical shorts inside the gas volume.

The gas mixture for the current measurement under high voltage is $95 \%$ tetrafluoroethane, $5 \%$ isobutane. The gas volume is purged by the flow of the gas mixture with flow rate of $20 \mathrm{l} / \mathrm{h}$, whose total volume is as large as 15 times the gas volume of the gas gap, before applying high voltage. The total duration of the current measurement is about 7 days including the purging time.

At the beginning of the test, the high voltage is set to $1 \mathrm{kV}$ to check for any electrical misconnection. If the gaps hold at $1 \mathrm{kV}$, then every 30 minutes, the $\mathrm{HV}$ is increased by $1 \mathrm{kV}$ up to $6 \mathrm{kV}$. At each HV setting, the currents are measured and recorded in the data sheet. From $6 \mathrm{kV}$ the $\mathrm{HV}$ is raised up to the maximum $10 \mathrm{kV}$. From $8.4 \mathrm{kV}$, the increasing step is $200 \mathrm{~V}$, finer than the $1 \mathrm{kV}$ step to see the current behaviors in the operation voltages. Once the HV has reached the 
maximum $10 \mathrm{kV}$, it is lowered to $9.6 \mathrm{kV}$ for a long-term test lasting 96 hours.

\section{Packing and transportation}

Every 60 qualified gas gaps are vertically mounted into a wooden box equipped with four pre-loaded bars which were designed to press the surface of the gas gaps horizontally. Polystyrene foam sheets of $5 \mathrm{~mm}$ thickness are placed between the layers of the gas gaps to absorb shocks which could occur during transportation. Extra protection from unexpected moisture is provided by wrapping the whole 60 gas gaps with a thin vinyl film. The pipes for the gas inlets and outlets are left open to adapt to sudden variations of pressure during air transport.

\section{Conclusions}

We report that the RPC production procedure and subsequent quality assurance procedure that Korea Detector Laboratory (KODEL) developed for the CMS forward RPC stations of RE1, RE2, RE3 are being followed to produce 660 gas gaps that will be assembled into RE4 station of the CMS forward regions.

We conclude that the production and subsequent quality assurance described here is mature for the mass production of the large RPC detectors for the CMS experiment.

\section{References}

[1] CMS Collaboration, The Muon Project, Technical Design Report, CERN/LHCC 97-32, December 1997.

[2] S. Park et al., Nucl. Instr. Meth. A 550 (2005) 551; Nucl. Instr. Meth. A 533 (2004) 37; Nucl. Instr. Meth. A 508 (2003) 147; Nucl. Instr. Meth. A 469 (2001) 323; Nucl. Instr. Meth. A 465 (2001) 447; Nucl. Instr. Meth. A 456 (2000) 29; Nucl. Instr. Meth. A 456 (2000) 23; Nucl. Instr. Meth. A 451 (2000) 582 .

[3] M. Tytgat, The Phase-1 Extension of the CMS Endcap Resistive Plate Chamber System, contribution to this proceedings. 